\title{
A Test for Risk-Averse Expected Utility
}

\author{
Christopher P. Chambers, Ce Liu, and Seung-Keun Martinez*
}

February 29, 2016

\begin{abstract}
We provide a universal condition for rationalizability by risk-averse expected utility preference in a demand-based framework with multiple commodities. Our test can be viewed as a natural counterpart of a classical test of expected utility, due to Fishburn (1975), in a demand setting.
\end{abstract}

\section{Introduction}

The recent contribution of Kubler et al. (2014) provides a GARP-like test for riskaverse expected utility maximization in a contingent-consumption environment. In an environment with a single consumption good and finite states of the world, they establish an acyclicity condition on observed data which is both necessary and sufficient for a finite list of observed price and consumption pairs to be consistent with the hypothesis of expected utility maximization. Thus, their paper provides a counterpart of the classical work of Afriat (1967) with the added restriction that rationalizations be risk-averse expected utility.

${ }^{*}$ Chambers, Liu, and Martinez are affiliated with the Department of Economics, University of California, San Diego. Emails: cpchambers@ucsd.edu (Chambers), cel013@ucsd.edu (Liu), and sem012@ucsd.edu (Martinez). Chambers gratefully acknowledges the support of the National Science Foundation through grant SES-1426867. We are grateful to Federico Echenique, John Quah and Marciano Siniscalchi for comments and suggestions. Two anonymous referees also provided comments and corrections which substantially improved the paper. All errors are our own. 
As Kubler et al. (2014) note, their test is universal in nature, removing all existential quantification. Their test amounts to verifying that the product of certain cycles of risk-neutral prices be bounded above by one. Our aim in this note is to provide a different universal test. Our test should be distinguished from the Kubler et al. (2014) test in three ways. First, it applies to any finite number of consumption goods, whereas the test of Kubler et al. (2014) only applies for a single consumption good. Secondly, our test is intimately tied to the classical von Neumann-Morgenstern axioms of expected utility theory, and thus has a simple economic intuition. On the other hand, our test involves universal quantification over a potentially infinite number of objects, while the test in Kubler et al. (2014) can be reduced to universal quantification over a finite set.

We emphasize that what we mean by test is a method for falsifying the model with directly observable data. In other words, we say a model is testable if whenever data are inconsistent with the model, they can be demonstrated to be inconsistent. In this sense of the term test, a demonstration is distinct from an algorithm which would find this falsifying certificate. Hence, a test in our sense is not intended to be useful from a computational perspective, and as far as we can tell, ours is not in general. Indeed; there are already practical algorithms for determining when the expected utility model is falsified in our context. Rather, such a test is important for understanding the economic content of the model, by specifying a condition stated in terms of data alone, which does not reference unobservable concepts such as utilities or marginal rates of substitution. As a point of comparison, the work of Richter (1966) can be understood as providing the testable restrictions of the preference maximization hypothesis; however, no general algorithm would exist in Richter's case either. ${ }^{1}$

Our test is perhaps most closely related to an early revealed preference test of expected utility due to Fishburn (1975). Fishburn constructs a test for an abstract environment of choice over lotteries with finite support. In his setting, one observes

\footnotetext{
${ }^{1}$ In the special case where budgets are given by linear inequalities and preference satisfies monotonicity, an algorithm exists for Richter's test, namely the Afriat test. Here we refer to the abstract budget environment.
} 
a finite set of binary comparisons; some are weak, and some are strict. Fishburn provides necessary and sufficient conditions for there to exist an expected utility ranking which extends the observed binary comparisons. Imagine that we observe lottery $l_{k}$ weakly preferred to lottery $l_{k}^{\prime}$ for $k=1, \ldots, g$, and $l_{k}$ strictly preferred to $l_{k}^{\prime}$ for $k=g+1, \ldots, K$. Fishburn establishes that these observations are consistent with expected utility maximization if there is no probability distribution over $\{1, \ldots, K\}$ which puts positive probability on $\{g+1, \ldots, K\}$, and for which the mixture of the $l_{k}$ 's under this probability distribution is equal to the mixture of the $l_{k}^{\prime}$ 's. Fishburn's test can be viewed as claiming that the smallest possible extension of the observed relations satisfying both independence and transitivity leads to no contradiction. We stress that Fishburn's test also presents with no algorithm: no recipe is given for finding the probability distribution.

In our case, we have $n$ commodities, and a finite set of states $\Omega=\{\omega \mid 1,2 \ldots, S\}$. We observe a finite list of prices and contingent consumption bundles chosen at those prices $\left(x^{k}, p^{k}\right), k \in\{1, \ldots K\}$. Consumption in state $\omega$ at observation $k$ is of the form $x_{\omega}^{k} \in \mathbb{R}_{+}^{n}$. Probabilities over $\Omega$ are known and are given by the full support distribution $\pi$.

We first ask: What could reveal a violation of the joint hypothesis of expected utility and risk aversion in this context? There are only a finite set of states of the world, with known probabilities, but if the choices were rationalizable by an expected utility preference, there would be a natural extension to a preference over the set of all simple lotteries. One such violation would look like the following: suppose that for each $x^{k}$, there is some $y^{k}$ which is feasible at prices $p^{k}$. In other words, the induced lottery $l_{x^{k}}$ is revealed preferred to the induced lottery $l_{y^{k}}$. And suppose that there is some $g$ for which $y^{g}$ is strictly cheaper than $x^{g}$ at prices $p^{g}$. In other words, the induced lottery $l_{x^{g}}$ is revealed strictly preferred to the induced lottery $l_{y^{g}}$. Now, suppose we can find, for each $k$, a lottery $l_{k}^{\prime}$ which is a mean-preserving spread of $l_{y^{k}}$. If the data were rationalizable by a risk-averse expected utility preference, the lottery $l_{x^{k}}$ would be preferred to $l_{k}^{\prime}$ for all $k$ (and $l_{x^{g}}$ would be strictly preferred to $\left.l_{g}^{\prime}\right)$.

We now have a set of $K$ pairs of lotteries $\left(l_{x^{k}}, l_{k}^{\prime}\right)$ which could be obtained in the 
preceding fashion. These data can be tested with Fishburn's condition. If, in fact, they violate Fishburn's condition, then we know that the original data cannot be expected utility rationalizable.

So far this is very simple. However, in the demand setting, for each observation $\left(p^{k}, x^{k}\right)$, there are usually infinitely many candidates for the above $y^{k}$, and for each $y^{k}$, an infinite number of possible mean-preserving spreads $l_{k}^{\prime}$. This would result in an infinite number of possible $\left\{\left(l_{x^{k}}, l_{k}^{\prime}\right)\right\}_{k=1}^{K}$ sets. While the Fishburn condition is sufficient to ensure each $\left\{\left(l_{x^{k}}, l_{k}^{\prime}\right)\right\}_{k=1}^{K}$ set has its own preference extension, it has nothing to say about whether or not there is a single preference extension for the infinitely many revealed preference relations.

In fact, what we show is the following: If the data are not risk-averse expected utility rationalizable, then there exists at least one set, $\left\{\left(l_{x^{k}}, l_{k}^{\prime}\right)\right\}_{k=1}^{K}$, as above, that violates Fishburn's condition. In addition, they can be chosen to violate Fishburn's condition in a very stark way: one must only test the uniform lottery over $\{1, \ldots, K\}$.

Moreover, the support of each $l_{k}^{\prime}$ can be chosen to consist only of consumption that was actually observed demanded at some state; i.e. the support can be chosen amongst elements of the form $x_{\omega}^{k}$. This resonates with the idea from Polisson et al. (2015), who observe that in order to rationalize data, it is both necessary and sufficient to maintain consistency on the set of minimally extended "imaginary" data, constructed from those actually observed. However, while Polisson et al. (2015) is concerned with developing Afriat-style algorithms (see Afriat (1967)) for testing decision models with money lotteries, our focus is developing universal statements about data from lotteries of general consumption bundles, which provides direct falsification of the expected utility model under risk aversion.

It is important to note that due to the infinite nature of our test, our contribution lies not in providing a procedure to be implemented to check actual data; for such a test, the readers are directed to the work by Green and Srivastava (1986). Instead, the main contribution of our test is that it extends the intuition of the Fishburn test to demand-based observations: whenever the smallest possible extension of the observed relations satisfying both independence and transitivity leads to no contradiction, the data are rationalizable by risk-averse expected utility preference. In addition, the test 
by Green and Srivastava involves theoretical objects that are not directly observable, while our conditions directly characterize exactly which types of data are ruled out by the hypothesis of expected utility maximization, and thus can be interpreted as its UNCAF axiomatization, when observations are made in a demand-based framework. ${ }^{2}$

The idea of the proof is remarkably simple, and is a simple restatement of the dual set of linear inequalities stemming from the Afriat-style inequalities of Green and Srivastava (1986) or Varian (1983).

A host of other interesting papers have recently studied choice data in the context of expected utility maximization. In particular, Echenique and Saito (2015) investigates the subjective expected utility version of the model, which forms a kind of analogue of the Kubler et al. (2014) test. It would be interesting to propose a test of our structure in the subjective expected utility framework. Epstein (2000) investigates the empirical content of the notion of probabilistic sophistication (due to Machina and Schmeidler (1992)), providing a test which can refute the hypothesis.

\section{The Model}

We assume that there is a finite state space $\Omega=\{\omega \mid 1,2, \ldots, S\}$ and a finite collection of consumption goods, labeled $1,2, \ldots, N$. The agent is given an objective probability distribution over states $\pi \in \Delta(\Omega)$, where for all $\omega \in \Omega, \operatorname{Pr}(\omega)=\pi_{\omega}>0$. An observation is a pair $(p, x)$, where $p \in \mathbb{R}_{++}^{S N}$ is a list of the prices of all $N$ consumption goods under all $S$ possible states, and $x \in \mathbb{R}_{+}^{S N}$ details the purchased amount of each consumption good under each state of the world. ${ }^{3}$ We assume that our data set $\mathcal{D}$ consists of a $K$ tuple of $(x, p)$ pairs, i.e. $\mathcal{D}=\left\{\left(x^{k}, p^{k}\right)_{k=1}^{K}\right\} . K$ is assumed finite.

\footnotetext{
${ }^{2} \mathrm{UNCAF}$ stands for universal negation of conjunction of atomic formulas. Chambers et al. (2014) demonstrate that theories which make no non-empirical predictions are exactly those which have UNCAF axiomatizations.

${ }^{3}$ As usual, $\mathbb{R}_{++}$denotes the positive reals, and $\mathbb{R}_{+}$the nonnegative reals.
} 
In particular,

$$
x^{k}=\left[\begin{array}{c}
x_{1}^{k} \\
\vdots \\
x_{\omega}^{k} \\
\vdots \\
x_{S}^{k}
\end{array}\right] p^{k}=\left[\begin{array}{c}
p_{1}^{k} \\
\vdots \\
p_{\omega}^{k} \\
\vdots \\
p_{S}^{k}
\end{array}\right]
$$

and

$$
x_{\omega}^{k}=\left[\begin{array}{c}
x_{\omega, 1}^{k} \\
\vdots \\
x_{\omega, N}^{k}
\end{array}\right] p_{\omega}^{k}=\left[\begin{array}{c}
p_{\omega, 1}^{k} \\
\vdots \\
p_{\omega, N}^{k}
\end{array}\right]
$$

where for all $\omega, k, n, x_{\omega, n}^{k} \geq 0$ and $p_{\omega, n}^{k}>0$. Each $x^{k}$ is referred to as a contingent consumption bundle, and $x_{\omega}^{k}$ a state-specific consumption bundle. We use $C=\mathbb{R}_{+}^{N S}$ to denote the set of all contingent consumption bundles.

We say that $\mathcal{D}$ is risk-averse expected utility rationalizable if there exists a concave, continuous, and increasing $u: \mathbb{R}_{+}^{N} \rightarrow \mathbb{R}$ for which for all $k, x^{k}$ solves

$$
\max _{x \in \mathbb{R}_{+}^{S N}} \sum_{\omega} \pi_{\omega} u\left(x_{\omega}\right)
$$

subject to $p^{k} \cdot x \leq p^{k} \cdot x^{k} \cdot 4$

Given a data set $\mathcal{D}$, we collect all the state-specific consumption bundles $x_{\omega}^{k}$ observed in the data:

$$
\mathcal{X}=\left\{x \in \mathbb{R}_{+}^{N} \mid x=x_{\omega}^{k} \text { for some } k \text { and } \omega \text { where }\left(x^{k}, p^{k}\right) \in \mathcal{D}\right\}
$$

Denote the set of all simple lotteries on $\mathbb{R}_{+}^{N}$ with finite support by $\Delta_{s}\left(\mathbb{R}_{+}^{N}\right)$. Denote the set of all lotteries on $\mathcal{X}$ by $\Delta(\mathcal{X})$. Note that $\Delta(\mathcal{X}) \subseteq \Delta_{s}\left(\mathbb{R}_{+}^{N}\right)$.

Any contingent consumption bundle $x^{k} \in C$ induces an element $l_{x^{k}} \in \Delta(\mathcal{X})$,

\footnotetext{
${ }^{4}$ We take increasing to mean that if $x \geq y$ and $x \neq y$, then $u(x)>u(y)$.
} 
which places probability $\pi_{\omega}$ on $x_{\omega}^{k}$. As such, a pair of revealed preference relations $\succeq^{C}$ and $\succ^{C}$ can be defined on $\Delta(\mathcal{X})$ :

For $x, y \in C, l_{x} \succeq^{C} l_{y}$ if $x=x^{k}$ for some $\left(x^{k}, p^{k}\right) \in \mathcal{D}$ and $p^{k} \cdot y \leq p^{k} \cdot x$. For $x, y \in C, l_{x} \succ^{C} l_{y}$ if $x=x^{k}$ for some $\left(x^{k}, p^{k}\right) \in \mathcal{D}$ and $p^{k} \cdot y<p^{k} \cdot x$. $\succeq^{C}$ is intended to represented a revealed weak preference and $\succ^{C}$ a revealed strict preference.

Moreover, to test the hypothesis of risk aversion, it is natural to extend the above revealed preference relations to $\Delta_{s}\left(\mathbb{R}_{+}^{N}\right)$. For example, suppose that $l_{x} \succeq^{C}$ $l_{y}$, and $l \in \Delta_{s}\left(\mathbb{R}_{+}^{N}\right)$ can be obtained by a sequence of mean-preserving spreads of $l_{y}{ }^{5}$ If our decision maker's behavior is consistent with risk-averse expected utility maximization, it follows that $l_{x}$ should also be preferred to $l$. These ideas motivate the following definitions.

For $l, l^{\prime} \in \Delta_{s}\left(\mathbb{R}_{+}^{N}\right), l \succeq^{m . p . s .} l^{\prime}$ if $l^{\prime}$ can be obtained by a series of mean-preserving spreads of $l$. Define the pair of binary relations $\succeq^{R}$ and $\succ^{R}$ on $\Delta_{s}\left(\mathbb{R}_{+}^{N}\right)$ by

$$
l \succeq^{R} l^{\prime \prime} \text { if there exists } l^{\prime} \text { such that } l \succeq^{C} l^{\prime} \succeq^{m \cdot p \cdot s .} l^{\prime \prime}
$$

and

$$
l \succ^{R} l^{\prime \prime} \text { if there exists } l^{\prime} \text { such that } l \succ^{C} l^{\prime} \succeq^{m \cdot p \cdot s .} l^{\prime \prime}
$$

If the agent's behavior is consistent with risk-averse expected utility maximization, the pair of relations $\succeq^{R}, \succ^{R}$ will necessarily satisfy Fishburn's condition on $\Delta_{s}\left(\mathbb{R}_{+}^{N}\right)$; i.e. if $l_{k} \succeq^{R} l_{k}^{\prime}$ for $k=1, \ldots, g$, and $l_{k} \succ^{R} l_{k}^{\prime}$ for $k=g+1, \ldots, K$, then there are no $\left\{\mu_{i}\right\}_{i=1}^{K} \subseteq \mathbb{R}_{+}^{K}$, with $\sum_{k=g+1}^{K} \mu_{k}>0$, and $\sum_{1}^{K} \mu_{k} l_{k}=\sum_{1}^{K} \mu_{k} l_{k}^{\prime}$. As we show in our main result, it turns out that a sufficient condition for the data $\mathcal{D}$ to conform with risk aversion and expected utility maximization is that the restriction of $\succeq^{R}, \succ^{R}$ to $\Delta(\mathcal{X})$ satisfies Fishburn's condition.

Theorem 1. For every data set $\mathcal{D}=\left\{\left(x^{k}, p^{k}\right)_{k=1}^{K}\right\}$, the following are equivalent:

I For any $\left\{l_{k}^{\prime}\right\}_{k=1}^{K} \subseteq \Delta(\mathcal{X})$ for which $l_{x^{k}} \succeq^{R} l_{k}^{\prime}$ for all $k$, there is no $\left\{\mu_{k}\right\}_{k=1}^{K} \subseteq \mathbb{R}_{+}^{K}$ for which $\sum_{\left\{k: l_{k} \succ{ }^{R} l_{k}^{\prime}\right\}} \mu_{k}>0$ and $\sum_{1}^{K} \mu_{k} l_{x^{k}}=\sum_{1}^{K} \mu_{k} l_{k}^{\prime}$.

\footnotetext{
${ }^{5}$ That is, if there exists a random variable $\epsilon$ such that $l \stackrel{d}{=} l_{y}+\epsilon$ with $E\left(\epsilon \mid l_{y}\right)=0$. " $\frac{d}{=}$ here means "has the same distribution as". See Rothschild and Stiglitz (1970) for more details.
} 
II Suppose that for each $k \in\{1, \ldots, K\}$ and $\omega \in \Omega, S_{\omega}^{k}:\{1, \ldots, K\} \times \Omega \rightarrow \mathbb{R}_{+}$ is a function, such that for all $k, \omega, \sum_{g, \tau} S_{\omega}^{k}(g, \tau)=\pi_{\omega}=\sum_{g, \tau} S_{\tau}^{g}(k, \omega)$. If, in addition, for all $k$,

$$
p^{k} \cdot x^{k} \geq p^{k} \cdot\left(\frac{\sum_{g} \sum_{\tau} S_{\omega}^{k}(g, \tau) x_{\tau}^{g}}{\pi_{\omega}}\right)_{\omega \in \Omega}
$$

then there is no $k$ for which $p^{k} \cdot x^{k}>p^{k} \cdot\left(\frac{\sum_{g} \sum_{\tau} S_{\omega}^{k}(g, \tau) x_{\tau}^{g}}{\pi_{\omega}}\right)_{\omega \in \Omega} \cdot{ }^{6}$

III For all $\omega, \tau \in \Omega$ and $k, g \in\{1, \ldots, K\}$ there exist $u_{w}^{k}, u_{\tau}^{g} \geq 0$ and $\lambda_{k}, \lambda_{g}>0$ s.t. $u_{\omega}^{k} \leq u_{\tau}^{g}+\lambda_{g} \frac{p_{\tau}^{g}}{\pi_{\tau}} \cdot\left(x_{\omega}^{k}-x_{\tau}^{g}\right)^{7}$

IV Data set $\mathcal{D}$ is risk-averse expected utility rationalizable.

Before proceeding, we comment on cases I and II, which are our contribution. Case I considers the smallest possible preference extension "consistent" with the data, risk-aversion, and the expected utility hypothesis. It claims that if this extension is meaningfully defined; in that we cannot derive that a lottery $l$ is strictly preferred to itself, then the data are expected utility rationalizable. Importantly, we only need to consider lotteries whose support are actual observed consumption bundles. This can be seen as a natural analogue of Fishburn's condition as applied to $l_{x^{k}}$ and $l_{k}^{\prime}$.

Case II demonstrates a dual system of linear inequalities to the inequalities of case III, which was derived previously by Green and Srivastava (1986). The interpretation of the terms $S_{\omega}^{k}$ is as a system of probability weights. To obtain some intuition on Case II, suppose that the inequalities therein are satisfied, then one can find a contradiction as follows: For each $k$, by demand behavior, the inequalities in

$$
6\left(\frac{\sum_{g} \sum_{\tau} S_{\omega}^{k}(g, \tau) x_{\tau}^{g}}{\pi_{\omega}}\right)_{\omega \in \Omega}=\left(\frac{\sum_{g} \sum_{\tau} S_{1}^{k}(g, \tau) x_{\tau}^{g}}{\pi_{1}}, \ldots, \frac{\sum_{g} \sum_{\tau} S_{S}^{k}(g, \tau) x_{\tau}^{g}}{\pi_{S}}\right) \text { i.e. } \frac{\sum_{g} \sum_{\tau} S_{\omega}^{k}(g, \tau) x_{\tau}^{g}}{\pi_{\omega}} \text { is the }
$$
consumption in state $\omega$.

${ }^{7}$ Green and Srivistava's proof of this statement assumes the non-emptyness of $u$ 's superdifferential over $\mathbb{R}_{+}^{n}$; however, it is easy to modify their proof even with empty superdifferential on the boundary. Essentially, whenever $x^{g}$ is known to be a utility maximizer, we can always find $\nabla u\left(x_{\tau}^{g}\right)$ in the superdifferential of $u$ for which $\nabla u\left(x_{\tau}^{g}\right)=\lambda_{g} \frac{p_{\tau}^{g}}{\pi_{\tau}}$ (see Theorem 28.3 in Rockafellar (1997)). So $u_{\omega}^{k} \leq u_{\tau}^{g}+\nabla u\left(x_{\tau}^{g}\right) \cdot\left(x_{\omega}^{k}-x_{\tau}^{g}\right)=u_{\tau}^{g}+\lambda_{g} \frac{p_{\tau}^{g}}{\pi_{\tau}} \cdot\left(x_{\omega}^{k}-x_{\tau}^{g}\right)$. 
Case II imply that the lottery $l_{y^{k}}$ induced by the contingent consumption bundle $\left(\frac{\sum_{g} \sum_{\tau} S_{\omega}^{k}(g, \tau) x_{\tau}^{g}}{\pi_{\omega}}\right)_{\omega \in \Omega}$ is revealed weakly worse than the lottery $l_{x^{k}}$ induced by $x^{k}$, with strict preference for at least one $k$. Observe that $l_{y^{k}}$ is a lottery that places probability $\pi_{\omega}$ on $\frac{\sum_{g} \sum_{\tau} S_{\omega}^{k}(g, \tau) x_{\tau}^{g}}{\pi_{\omega}}$. Since $\sum_{g, \tau} S_{\omega}^{k}(g, \tau)=\pi_{\omega}$, simple algebra (included in the proof) shows that the lottery $l_{k}^{\prime}$, which places probability weight $S_{\omega}^{k}(g, \tau)$ on $x_{\tau}^{g}$, is a mean-preserving spread of $l_{y^{k}}$. If the data were really consistent with the hypothesis of risk-averse expected utility maximization, transitivity would imply that for each $k$, the lottery $l_{k}^{\prime}$ should be worse than the lottery $l_{x^{k}}$, strictly so for at least one $k$. We now have in total $K$ revealed preference relations between the pairs of lotteries $l_{x^{k}}$ and $l_{k}^{\prime}$. As we demonstrate in the proof, the condition $\sum_{g, \tau} S_{\tau}^{g}(k, \omega)=\pi_{\omega}$ then allows us to find a violation by applying the condition from Fishburn (1975) on the lotteries $l_{x^{k}}$ and $l_{k}^{\prime}$ across all $k$.

The following example illustrates the theorem.

Example 1. Consider the case $k \in\{1,2\}, \Omega=\{1,2\}$ and $N=2$ : There are 2 observations, each consisting of the price and purchased quantity for the consumptions good under 2 possible states of the world. Suppose each of the two states are equally likely; $\pi_{1}=\pi_{2}=.5$. Suppose we observe:

$$
\left(x^{1}, p^{1}\right)=\left(\left[\begin{array}{c}
0 \\
0 \\
10 \\
5
\end{array}\right],\left[\begin{array}{c}
5 \\
10 \\
5 \\
10
\end{array}\right]\right)\left(x^{2}, p^{2}\right)=\left(\left[\begin{array}{c}
4 \\
2 \\
6 \\
3
\end{array}\right],\left[\begin{array}{c}
4 \\
8 \\
5 \\
10
\end{array}\right]\right)
$$

In this case there is no violation of GARP. However, since the hypothesis of risk-averse EU preference is stronger than than GARP, we show that this case still violates our conditions.

Violation of Statement I: The induced lotteries by $x^{1}$ and $x^{2}$ are $l_{x^{1}}=$ $((10,5), 1 / 2 ;(0,0), 1 / 2)$ and $l_{x^{2}}=((4,2), 1 / 2 ;(6,3), 1 / 2)$, respectively. To see that this is a violation of statement $I$, consider contingent consumption bundles $y^{1}=$ 
$y^{2}=((5,2.5) ;(5,2.5))$ which induce $l_{y^{1}}=l_{y^{2}}=((5,2.5), 1)$. Clearly $p^{1} \cdot x^{1} \geq p^{1} \cdot y^{1}$, and $p^{2} \cdot x^{2}>p^{2} \cdot y^{2}$. So by definition $l_{x^{1}} \succeq^{C} l_{y^{1}}$ and $l_{x^{2}} \succ^{C} l_{y^{2}}$.

Observe that the lottery $l_{1}^{\prime}=((4,2), 1 / 2 ;(6,3), 1 / 2)$ is a mean-preserving spread of $l_{y^{1}}$ and the lottery $l_{2}^{\prime}=((10,5), 1 / 2 ;(0,0), 1 / 2)$ is a mean-preserving spread of $l_{y^{2}}$. By definition $l_{x^{1}} \succeq^{R} l_{1}^{\prime}$ and $l_{x^{2}} \succ^{R} l_{2}^{\prime}$. However,

$$
\frac{1}{2} l_{x^{1}}+\frac{1}{2} l_{x^{2}}=\frac{1}{2} l_{1}^{\prime}+\frac{1}{2} l_{2}^{\prime}
$$

This constitutes a violation of Statement I.

\section{Violation of Statement II:}

Set $S_{1}^{1}(2,1)=S_{1}^{2}(1,1)=\frac{1}{5}, S_{1}^{1}(2,2)=S_{2}^{2}(1,1)=\frac{3}{10}, S_{2}^{1}(2,1)=S_{1}^{2}(1,2)=\frac{3}{7}$, and $S_{2}^{1}(2,2)=S_{2}^{2}(1,2)=\frac{1}{14}$.

To solve:

$$
\left[\begin{array}{c}
0 \\
0 \\
10 \\
5
\end{array}\right] \cdot\left[\begin{array}{c}
5 \\
10 \\
5 \\
10
\end{array}\right]>2 \cdot\left[\begin{array}{c}
5 \\
10 \\
5 \\
10
\end{array}\right] \cdot\left[\begin{array}{l}
S_{1}^{1}(2,1) * 4+S_{1}^{1}(2,2) * 6 \\
S_{1}^{1}(2,1) * 2+S_{1}^{1}(2,2) * 3 \\
S_{2}^{1}(2,1) * 4+S_{2}^{1}(2,2) * 6 \\
S_{2}^{1}(2,1) * 2+S_{2}^{1}(2,2) * 3
\end{array}\right]
$$

$$
\begin{gathered}
{\left[\begin{array}{l}
4 \\
2 \\
6 \\
3
\end{array}\right] \cdot\left[\begin{array}{c}
4 \\
8 \\
5 \\
10
\end{array}\right] \geq 2 \cdot\left[\begin{array}{c}
4 \\
8 \\
5 \\
10
\end{array}\right] \cdot\left[\begin{array}{c}
S_{1}^{2}(1,1) * 0+S_{1}^{2}(1,2) * 10 \\
S_{1}^{2}(1,1) * 0+S_{1}^{2}(1,2) * 5 \\
S_{2}^{2}(1,1) * 0+S_{2}^{2}(1,2) * 10 \\
S_{2}^{2}(1,1) * 0+S_{2}^{2}(1,2) * 5
\end{array}\right]} \\
S_{1}^{1}(2,1)+S_{1}^{1}(2,2)=S_{2}^{1}(2,1)+S_{2}^{1}(2,2)=\frac{1}{2}
\end{gathered}
$$


A couple of observations are in order. It can be shown that both (I) and (II) of our properties imply GARP. Suppose by means of contradiction that GARP is violated, i.e. that there are contingent consumption bundles $z^{k_{1}}, \ldots, z^{k_{m}}$ such that $p^{k_{1}} \cdot z^{k_{1}} \geq p^{k_{1}} \cdot z^{k_{2}}, p^{k_{2}} \cdot z^{k_{2}} \geq p^{k_{2}} \cdot z^{k_{3}}, \ldots, p^{k_{m}} \cdot z^{k_{m}}>p^{k_{m}} \cdot z^{k_{1}}$, where without loss we may assume there is no repetition in the cycle. This implies $l_{z^{k_{1}}} \succeq^{C} l_{z^{k_{2}}} \succeq^{C} \ldots \succeq^{C}$ $l_{z^{k_{m}}} \succ^{C} l_{z^{k_{1}}}$.

To see that (I) implies GARP, observe that since $\succeq^{C}$ implies $\succeq^{R}$ and $\succ^{C}$ implies $\succ^{R}$, we have $l_{z^{k_{1}}} \succeq^{R} l_{z^{k_{2}}} \succeq^{R} \ldots l_{z^{k_{m}}} \succ^{R} l_{z^{k_{1}}}$. Let $l_{x^{i}}=l_{z^{k_{i}}}$ and $l_{i}^{\prime}=l_{z^{k_{i+1}}}$ as in property (I), then a uniform distribution $\mu$ over the indices $i=1,2, \ldots m$ constitutes a violation of (I).

For (II), consider the following set of $S_{\omega}^{k}(g, \tau)$ 's in property II: For $k=k_{i}$ for some $i$ (that is, if $k$ shows up in the cycle)

$$
S_{\omega}^{k_{i}}(g, \tau)= \begin{cases}\pi_{\omega} & \text { if } g=k_{i+1} \text { and } \tau=\omega \\ 0 & \text { otherwise }\end{cases}
$$

and for $k \neq k_{i}$ for any $i(k$ not in the cycle)

$$
S_{\omega}^{k}(g, \tau)= \begin{cases}\pi_{\omega} & \text { if } g=k \text { and } \tau=\omega \\ 0 & \text { otherwise }\end{cases}
$$

Then the cycle condition gives a violation of property (II), a contradiction.

Finally, we wish to emphasize that the result is by no means a trivial consequence of Fishburn (1975). In his paper, he also considers the issue of testing the consistency of revealed preference relations with functional restrictions on the von Neumann-Morgenstern utility index (as we wish to test for concavity and monotonicity). Specifically, he wants to test when observed data are consistent with the utility index $u$ belonging to some convex cone $\mathcal{U}$. Again, he assumes a finite number of relations (which does not hold in our context). A natural guess is that if $l_{k}$ is revealed weakly preferred to $l_{k}^{\prime}$ for $k=1, \ldots, g$ and revealed strictly preferred to $l_{k}^{\prime}$ for $k=g+1, \ldots, K$, then if there is $\mu \in \Delta(K)$ for which $\mu(\{g+1, \ldots, K\})>0$ and 
$u \cdot\left(\sum_{k} \mu_{k} l_{k}^{\prime}\right) \geq u \cdot\left(\sum_{k} \mu_{k} l_{k}\right)$ for all $u \in \mathcal{U}$, then the observed data are inconsistent with expected utility maximization with utility index $u \in \mathcal{U}^{8}$. In our case, for example, we would consider the cone of concave, nondecreasing and locally non-satiated functions; the claim would then be that $\sum_{k} \mu_{k} l_{k}^{\prime}$ second order stochastically dominates $\sum_{k} \mu_{k} l_{k}$. Of course, the existence of such a $\mu$ refutes the hypothesis of expected utility rationalization with $u \in \mathcal{U}$, but for technical reasons, the converse statement need not hold in general (it would hold, for example, if the cone $\mathcal{U}$ were polyhedral, which is not the case here). However, we are able to show that owing to the special structure of linear pricing, a converse statement along the lines of this idea does in fact hold in the demand-based environment. In fact, it holds even though observed revealed preference relations are infinite.

Proof. (III $\Leftrightarrow$ IV)

The equivalence of III and IV is due to Green and Srivastava (1986).

\section{$(\mathrm{II} \Leftrightarrow \mathrm{III})$}

We proceed to show that II and III are equivalent. To this end, observe that III does not hold if and only if there is no solution to the following linear system. ${ }^{9} A b \geq 0$ and $\lambda \gg 0$, where

$$
b=\left[\begin{array}{c}
u_{1}^{1} \\
u_{2}^{1} \\
\vdots \\
u_{S}^{K} \\
\lambda
\end{array}\right] \lambda=\left[\begin{array}{c}
\lambda_{1} \\
\vdots \\
\lambda_{K}
\end{array}\right]
$$

and $A$ is equal to the top two quadrants of the matrix below:

\footnotetext{
${ }^{8}$ Here we continue to use $x$ and $z$ for lotteries, and dot product for integration with respect to measures.

${ }^{9}$ Vector inequalities are $x \geq y$ if $x_{i} \geq y_{i}$ for all $i$ and $x \gg y$ if $x_{i}>y_{i}$ for all $i$.
} 


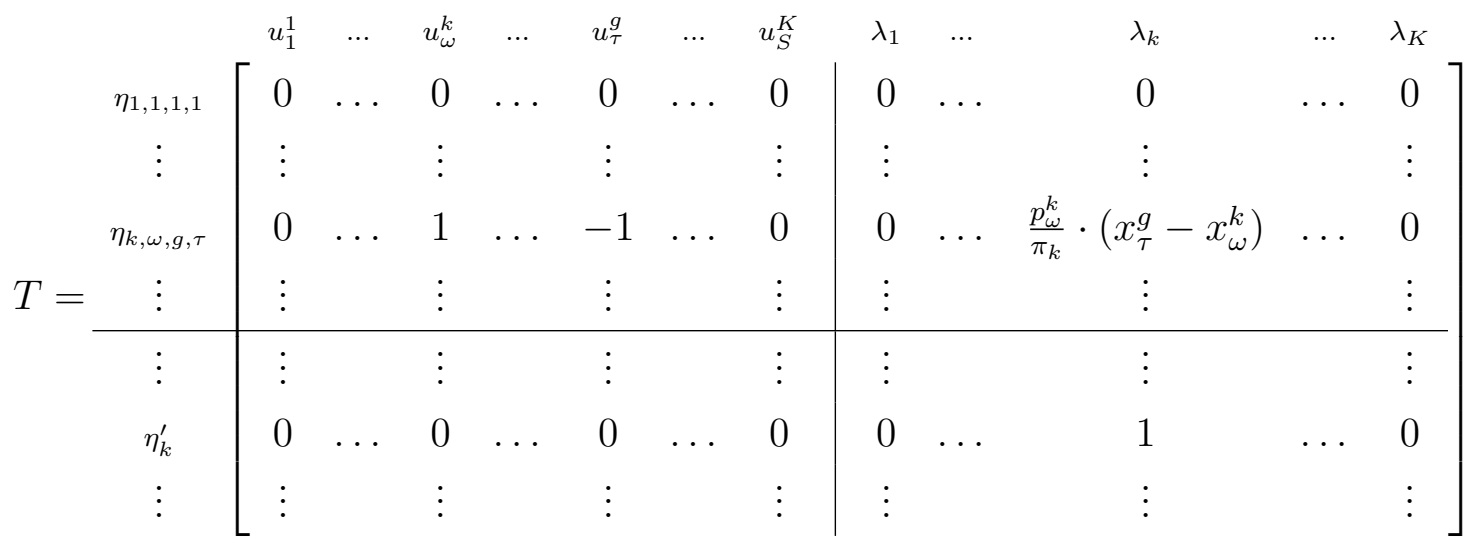

By construction of $T$ and a standard theorem of the alternative (see for example Mangasarian (1994) p. 30), the nonexistence of $b, \lambda$ such that $A b \geq 0$ and $\lambda \gg 0$, is equivalent to the existence of $\eta \geq 0$ such that $T^{\prime} \eta \leq 0$, where

$$
\eta=\left[\begin{array}{c}
\eta_{1,1,1,1} \\
\vdots \\
\eta_{K, S, K, S} \\
\eta^{\prime}
\end{array}\right] \eta^{\prime}=\left[\begin{array}{c}
\eta_{1}^{\prime} \\
\vdots \\
\eta_{K}^{\prime}
\end{array}\right]
$$

such that at least one $\eta_{k}^{\prime}>0$.

This is equivalent to

$$
\sum_{\omega} \sum_{(g, \tau) \neq(k, \omega)} \eta_{k, \omega, g, \tau} \frac{p_{\omega}^{k}}{\pi_{\omega}} \cdot\left(x_{\tau}^{g}-x_{\omega}^{k}\right) \leq 0 \forall k
$$

with strict inequality for at least one $k$, and

$$
\sum_{(g, \tau) \neq(k, \omega)} \eta_{k, \omega, g, \tau}=\sum_{(g, \tau) \neq(k, \omega)} \eta_{g, \tau, k, \omega} \forall k, \omega
$$


We claim that a solution to systems (1) and (2), implies the existence of $\gamma_{k, \omega, g, \tau} \geq$ 0 so that

$$
\begin{gathered}
\sum_{\omega} \sum_{(g, \tau)} \gamma_{k, \omega, g, \tau} \frac{p_{\omega}^{k}}{\pi_{\omega}} \cdot\left(x_{\tau}^{g}-x_{\omega}^{k}\right) \leq 0 \forall k \\
\sum_{(g, \tau)} \gamma_{k, \omega, g, \tau}=\sum_{(g, \tau)} \gamma_{g, \tau, k, \omega}=\pi_{\omega} \forall k, \omega
\end{gathered}
$$

with at least one inequality in (3) being strict, effectively showing (3) and (4) are equivalent to (1) and (2).

To see this, list the $\eta_{k, \omega, g, \tau}$ 's from systems (1) and (2) as in Figure 1 (Notice that system (2) ensures that columns and rows passing through the same diagonal element, like the column and row in red and blue boxes, sum up to the same number.) We now construct a new matrix, say, $\Lambda$, with generic element $\lambda_{k, \omega, g, \tau}$ by raising all diagonal entries of the $\eta$ matrix, leaving all remaining entries the same, so that there is some $M>0$ for which $\sum_{(g, \tau)} \eta_{k, \omega, g, \tau}=\sum_{(g, \tau)} \eta_{g, \tau, k, \omega}=M \pi_{\omega} \cdot{ }^{10}$ Since $p_{\omega}^{k} \cdot\left(x_{\omega}^{k}-x_{\omega}^{k}\right)=0$, and since the diagonal element shows up both in the column and and row, the resulting $\eta$ matrix satisfies (3) (with $\lambda$ 's in place of $\eta$ 's), and the first equality in system (4). Finally, the $\gamma$ terms are constructed by dividing each element of the matrix $\Lambda$ by $M$.

Rearranging inequalities (3) gives

$$
\begin{gathered}
\sum_{\omega} \sum_{g} \sum_{\tau} \gamma_{k, \omega, g, \tau} \frac{p_{\omega}^{k}}{\pi_{\omega}} \cdot\left(x_{\tau}^{g}-x_{\omega}^{k}\right)=\sum_{\omega} p_{\omega}^{k} \cdot\left(\sum_{g} \sum_{\tau} \frac{\gamma_{k, \omega, g, \tau} x_{\tau}^{g}}{\pi_{\omega}}-\sum_{g} \sum_{\tau} \frac{\gamma_{k, \omega, g, \tau} x_{\omega}^{k}}{\pi_{\omega}}\right) \\
=\sum_{\omega} p_{\omega}^{k} \cdot\left(\sum_{g} \sum_{\tau} \frac{\gamma_{k, \omega, g, \tau} x_{\tau}^{g}}{\pi_{\omega}}-x_{\omega}^{k}\right) \leq 0
\end{gathered}
$$

with at least one strict inequality. The second equality follows from (4). This together with (4) establishes the equivalence of II and III, by taking $S_{\omega}^{k}(g, \tau)=\gamma_{k, \omega, g, \tau}$.

\footnotetext{
${ }^{10}$ One simple way of doing this is to pick $M$ large enough so that $\min _{\omega} \pi_{\omega} M>$ $\max _{\omega} \sum_{(g, \tau) \neq(k, \omega)} \eta_{k, \omega, g, \tau}$.
} 


1,1
$k, \omega$$\left[\begin{array}{cc|c|cccccc}1,1 & \multicolumn{1}{c}{1, \omega} & & 1, S & & k, \omega & & K, S \\ \eta_{1,1,1,1} & \cdots & \eta_{1,1,1, \omega} & \cdots & \eta_{1,1,1, S} & \cdots & \eta_{1,1, k, \omega} & \cdots & \eta_{1,1, K, S} \\ \vdots & \ddots & \vdots & \ddots & \vdots & \ddots & \vdots & \ddots & \cdots \\ \hline \eta_{1, \omega, 1,1} & \cdots & \eta_{1, \omega, 1, \omega} & \cdots & \eta_{1, \omega, 1, S} & \cdots & \eta_{1, \omega, k, \omega} & \cdots & \eta_{1, \omega, K, S} \\ \hline \vdots & \ddots & \vdots & \ddots & \vdots & \ddots & \vdots & \ddots & \cdots \\ \eta_{1, S, 1,1} & \cdots & \eta_{1, S, 1, \omega} & \cdots & \eta_{1, S, 1, S} & \cdots & \eta_{1, S, k, \omega} & \cdots & \eta_{1, S, K, S} \\ \vdots & \ddots & \vdots & \ddots & \vdots & \ddots & \vdots & \ddots & \cdots \\ \eta_{k, \omega, 1,1} & \cdots & \eta_{k, \omega, 1, \omega} & \cdots & \eta_{k, \omega, 1, S} & \cdots & \eta_{k, \omega, k, \omega} & \cdots & \eta_{k, \omega, K, S} \\ \vdots & \ddots & \vdots & \ddots & \vdots & \ddots & \vdots & \ddots & \cdots \\ \eta_{K, S, 1,1} & \cdots & \eta_{K, S, 1, \omega} & \cdots & \eta_{K, S, 1, S} & \cdots & \eta_{K, S, k, \omega} & \cdots & \eta_{K, S, K, S}\end{array}\right]$

Figure 1: $\eta$ matrix

\section{$(\mathrm{IV} \Rightarrow \mathrm{I})$}

That IV implies I is straightforward. Let $u: \mathbb{R}_{+}^{N} \rightarrow \mathbb{R}$ be any concave, nondecreasing and locally non-satiated utility function. For lottery $l$, let $u \cdot l$ denote the expected utility of $l, \sum_{x \in l} l(x) u(x)$.

Suppose that $\mathcal{D}$ is risk-averse expected utility rationalizable by $u$, and suppose by means of contradiction that statement $\mathrm{I}$ is not true

For all $l_{k}^{\prime} \in \Delta_{s}\left(\mathbb{R}_{+}^{N}\right), l_{x^{k}} \succeq^{R} l_{k}^{\prime}$ implies $u \cdots l_{x^{k}} \geq u \cdot l_{k}^{\prime}$, and $l_{x^{k}} \succ^{R} l_{k}^{\prime}$ implies $u \cdot l_{x^{k}}>u \cdot l_{k}^{\prime}$. Since expected utility is linear in lottery mixtures, we have that $u \cdot\left(\sum_{1}^{K} \mu_{k} l_{x^{k}}\right)>u \cdot\left(\sum_{1}^{K} \mu_{k} l_{k}^{\prime}\right)$, a contradiction to $\sum_{1}^{K} \mu_{k} l_{x^{k}}=\sum_{1}^{K} \mu_{k} l_{k}^{\prime}$.

\section{$(\mathrm{I} \Rightarrow \mathrm{II})$}

We now show that I implies II. Suppose by means of contradiction that there is a solution to the system listed in II. We will show that this implies I is false. Let

$$
y^{k}=\left(\frac{\sum_{g} \sum_{\tau} S_{\omega}^{k}(g, \tau) x_{\tau}^{g}}{\pi_{\omega}}\right)_{\omega \in \Omega}
$$


By II, we have $p^{k} \cdot x^{k} \geq p^{k} \cdot y^{k} \forall k$ with $>$ for at least one $k$. By definition of $\succeq^{C}$, $l_{x^{k}} \succeq^{C} l_{y^{k}}$, with $\succ^{C}$ for at least one $k$.

Next, observe that $l_{y^{k}}$ places probability $\pi_{\omega}$ at $\frac{\sum_{g} \sum_{\tau} S_{\omega}^{k}(g, \tau) x_{\tau}^{g}}{\pi_{\omega}}$ for each $\omega$. Let $l_{k}^{\prime}$ be the lottery that puts probability $\sum_{\omega} S_{\omega}^{k}(g, \tau)$ on $x_{\tau}^{g}$. Since $\sum_{g, \tau} S_{\omega}^{k}(g, \tau)=\pi_{\omega}$, $l_{k}^{\prime}$ can be obtained from $l_{y^{k}}$ by spreading, for each $\omega$, the probability $\pi_{\omega}$ placed on $\frac{\sum_{g} \sum_{\tau} S_{\omega}^{k}(g, \tau) x_{\tau}^{g}}{\pi_{\omega}}$ to probabilities $S_{\omega}^{k}(g, \tau)$ 's on $x_{\tau}^{g}$ 's, $(g, \tau) \in\{1, \cdots, K\} \times \Omega$. Moreover, $\frac{\sum_{g} \sum_{\tau} S_{\omega}^{k}(g, \tau) x_{\tau}^{g}}{\pi_{\omega}}$ is a weighted average of the $x_{\tau}^{g}$ 's by weights $S_{\omega}^{k}(g, \tau)$ 's. So for each $\omega$ the spread described above is a mean-preserving spread in the sense of Rothschild and Stiglitz (1970), and $l_{k}^{\prime}$ can be obtained from $l_{y^{k}}$ by a finite number of mean-preserving spread.

By definition of $\succeq^{R}$, we have obtained lotteries $l_{x^{k}}$ and $l_{k}^{\prime}$ such that $l_{x^{k}} \succeq^{R} l_{k}^{\prime} \forall k$, with $\succ^{R}$ for at least one $k$. In order to contradict I, it only remains now to find $\left\{\mu_{k}\right\}_{k=1}^{K}$ such that $\mu_{k} \geq 0, \sum_{\left\{k: l_{k} \succ R l_{k}^{\prime}\right\}} \mu_{k}>0$ and $\sum_{1}^{K} \mu_{k} l_{x^{k}}=\sum_{1}^{K} \mu_{k} l_{k}^{\prime}$. As it turns out, it suffices to take $\mu_{k}=\frac{1}{K}$ for each $k$ :

The lottery $\sum_{k=1}^{K} \frac{1}{K} l_{k}^{\prime}$ places probability $\frac{1}{K} \sum_{k} \sum_{\omega} S_{\omega}^{k}(g, \tau)=\frac{\pi_{\tau}}{K}$ on each $x_{\tau}^{g}$, $(g, \tau) \in\{1, \cdots, K\} \times \Omega$, while the lottery $\sum_{k=1}^{K} \frac{1}{K} l_{x^{k}}$, places $\frac{\pi_{\tau}}{K}$ on each $x_{\tau}^{g}$. So $\sum_{k=1}^{K} \frac{1}{K} l_{k}^{\prime}=\sum_{k=1}^{K} \frac{1}{K} l_{x^{k}}$. This constitutes a contradiction to I (in particular, the contradiction comes in the form of a uniform distribution over the observations $1, \ldots, K)$.

\section{Conclusion}

We have developed a universal test for the risk-averse expected utility environment with many commodities. Of interest for future research would be an analogous test in the subjective expected utility context, following the work of Echenique and Saito (2015). The difficulty inherent in this approach rests in the fact that the inequalities in III of Theorem 1 are polynomial, rather than linear. While we have some conjectures on what might be an appropriate test, these are very speculative.

A final remark is in order. Observe that when $|\Omega|=1$ (and hence $\pi_{\omega}=1$ for $\omega$ for which $\Omega=\{\omega\}$ ), we are back to the environment of Afriat (1967). In 
such an environment, the function $S$ referenced in Theorem 1, condition II can be taken to be a function of $\{1, \ldots, K\}$ alone. And condition II in this case tells us that $\sum_{k} S_{k}(l)=\sum_{k} S_{l}(k)=1$ for each $l$; in other words, viewing $S$ as a matrix, the matrix is bistochastic. Now, one of the contributions of Afriat (1967) is that condition II is necessary and sufficient for concave rationalization when the matrix $S$ is restricted to be a permutation matrix; that is, a matrix consisting solely of zeroes and ones. Of course, it is well-known that the permutation matrices are the extreme points of the set of bistochastic matrices (this is the celebrated theorem of Birkhoff (1946) and von Neumann (1953)). A natural conjecture is that a similar statement may hold here; that it is enough to check the extreme points of the set of $S$ functions satisfying condition II of Theorem 1.

\section{References}

Sidney N. Afriat. The construction of utility functions from expenditure data. International economic review, 8(1):67-77, 1967.

Garrett Birkhoff. Tres observaciones sobre el álgebra lineal. Univ. Nac. Tucumán Rev. Ser. A, 5:147-151, 1946.

Christopher P. Chambers, Federico Echenique, and Eran Shmaya. The axiomatic structure of empirical content. American Economic Review, 104(8):2303-2319, 2014.

Federico Echenique and Kota Saito. Savage in the market. Econometrica, 83(4): 1467-1495, 2015.

Larry G. Epstein. Are probabilities used in markets? Journal of Economic Theory, 91(1):86-90, 2000.

Peter C. Fishburn. Separation theorems and expected utilities. Journal of Economic Theory, 11(1):16-34, 1975. 
Richard C. Green and Sanjay Srivastava. Expected utility maximization and demand behavior. Journal of Economic Theory, 38(2):313-323, 1986.

Felix Kubler, Larry Selden, and Xiao Wei. Asset demand based tests of expected utility maximization. American Economic Review, 104(11):3459-3480, 2014.

Mark J. Machina and David Schmeidler. A more robust definition of subjective probability. Econometrica, 60(4):745-780, 1992.

Olvi L. Mangasarian. Nonlinear Programming. Classics in Applied Mathematics. Society for Industrial and Applied Mathematics, 1994. ISBN 9780898713411.

Matthew Polisson, Ludovic Renou, and John Quah. Revealed preference tests over risk and uncertainty. working paper, 2015.

Marcel K. Richter. Revealed preference theory. Econometrica, 34(3):635-645, 1966.

Tyrrell Rockafellar. Convex Analysis. Number 28. Princeton University Press, 1997.

Michael Rothschild and Joseph E. Stiglitz. Increasing risk: I. a definition. Journal of Economic theory, 2(3):225-243, 1970.

Hal R. Varian. Nonparametric tests of models of investor behavior. Journal of Financial and Quantitative Analysis, 18(3):269-278, 1983.

John von Neumann. A certain zero-sum two-person game equivalent to the optimal assignment problem. Contributions to the Theory of Games, 2:5-12, 1953. 